\title{
Transatlantica
}

Revue d'études américaines. American Studies Journal

$2 \mid 2017$

(Hi)stories of American Women: Writings and Rewritings / Call and Answer: Dialoguing the American West in France

\section{The sequoia files : L'arbre merveilleux}

\section{François Brunet}

\section{OpenEdition}

\section{Journals}

Édition électronique

URL : https://journals.openedition.org/transatlantica/9881

DOI : 10.4000/transatlantica.9881

ISSN : 1765-2766

Éditeur

Association française d'Etudes Américaines (AFEA)

Référence électronique

François Brunet, «The sequoia files : L'arbre merveilleux », Transatlantica [En ligne], 2 | 2017, mis en ligne le 19 mai 2019, consulté le 02 février 2023. URL : http://journals.openedition.org/transatlantica/ 9881 ; DOI : https://doi.org/10.4000/transatlantica.9881

Ce document a été généré automatiquement le 2 février 2023

\section{c) (i) $($ )}

Creative Commons - Attribution - Pas d'Utilisation Commerciale - Pas de Modification 4.0 International - CC BY-NC-ND 4.0

https://creativecommons.org/licenses/by-nc-nd/4.0/ 


\title{
The sequoia files : L'arbre merveilleux
}

\author{
François Brunet
}

Première publication dans Cahiers Charles V n²5, p. 77-96. Reproduit avec l'aimable autorisation de Catherine Bernard (Université Paris-Diderot).

1 En 1854 était exposée au Crystal Palace de New York une hauteur d'écorce de plus de cent pieds, provenant d'un «Tree Mastodon » de la Sierra Nevada. Dans les décennies qui suivirent, alors que l'industrie forestière exploitait un filon plus durable que l'or, le «Big Tree » de Californie, localement dénommé redwood, ailleurs séquoïa, devint un phénomène culturel. Dès 1864, le bosquet de Mariposa était intégré avec la vallée voisine de Yosemite au premier parc naturel américain. Trente ans plus tard, lors de l'Exposition Colombienne de Chicago en 1893, le séquoïa était encore chose assez remarquable pour que les commissaires fassent abattre un spécimen de trois cents pieds de hauteur - alors même que trois nouveaux parcs nationaux venaient d'être créés en Californie autour des principaux bosquets. S'il y eut, comme le montre Nancy K. Anderson, bien des contradictions dans la promotion du séquoïa (Anderson, 274), on retiendra surtout ici un processus durable de monumentalisation du grand arbre.

Géant et ancêtre de la forêt, le séquoïa devint en effet très vite le monument américain par excellence, et un emblème fétiche du courant primitiviste et nativiste. Simon Schama a brillamment montré que cette lecture emblématique, nourrie de tout un arrière-plan romantique et biblique, prospéra surtout dans le contexte de la guerre de Sécession, où le séquoïa fournit un symbole bienvenu à l'idéologie nationaliste ; grâce aux efforts d'un petit groupe d'intellectuels réunis par leur tropisme san-franciscain ${ }^{1}$, l'antiquité et l'immensité de l'arbre, réveillant l'image romantique de la cathédrale naturelle apparurent avec les falaises de Yosemite voisines comme "une révélation irrésistible du caractère unique de la République américaine ", justifiant la création du parc californien (Schama, 194-197). Cette révélation devait s'imposer bientôt à John Muir, futur inspirateur du mouvement « conservationniste ».

Mais parallèlement à ce culte aux accents genteel, le séquoïa séduisit aussi un lyrisme de la Frontière moins curieux des arbres que des hommes qui les abattaient. On pense ici à 
Walt Whitman, dont le célèbre poème "Song of the Redwood-Tree » (1874), donnant la parole à un redwood mourant, valait en réalité ode à l'Américain. En offrant sa vigueur éternelle en sacrifice au «new, culminating man » qui serait son héritier, le redwood devenait modèle d'américanité. Whitman communiait ici avec l'esprit de la Frontière, qui, comme l'avait vu Tocqueville quarante ans plus tôt, admirait moins la Nature que ses propres victoires sur celle-ci. Inscrivant « le génie du moderne » sur les ruines du séquoïa, il muait celui-ci en origine du geste pionnier; en préfigurait l'herméneutique turnérienne - laquelle ne célébra pas l'arbre, grand ou petit, mais plutôt la log-cabin du pionnier, qui, comme l'écrit Richard White, avait été au XIx siècle «l'icône principale de la frontière » (White, 19-21).

4 Malgré leurs divergences, ces deux modes d'assomption valaient donc " américanisation » du séquoïa, et participaient par là de ce qu'Angela Miller appelle "la dérégionalisation de l'Ouest», c'est-à-dire d'un travail idéologique des élites nationales pour effacer les différences régionales au profit «du statut national, historique " de l'Ouest (Miller, 128-133). Or, nous voudrions montrer ici que cette lecture idéologico-héroïque du séquoïa ne rend pas compte à elle seule de sa prodigieuse fécondité littéraire. De 1855 à 1900, ce sont des dizaines de relations de voyage en Californie et dans l'Ouest, américaines et européennes, illustrées ou non, et le plus souvent destinées au grand public, qui déclinèrent sur tous les tons l'émerveillement de l'observateur devant ce monstre de la nature. Cette littérature populaire, dont l'importance a été soulignée par Francis P. Farquhar ,reflète globalement le goût des «merveilles de l'Ouest » qui marqua les débuts du tourisme. Nous l'envisageons ici comme exemple privilégié d'un merveilleux naturel, d'un «pays des merveilles » réalisé dans le paysage californien - non pas tant par conséquent comme frontière américaine, mais comme frontière rhétorique, enracinée dans le mirage californien.

\section{De big bonanza à Big Tree : les séquoïas, rejetons de l'Eldorado}

5 L'Eldorado ou le "rêve californien " fut à bien des égards le redoublement du rêve américain: une Amérique au carré, irrésistible et insaisissable². L'identité ou plutôt l'image culturelle de cette frontière "ultime » et inversée - car orientée vers l'Est - dut beaucoup aux voyageurs, et à la rhétorique de l'émerveillement. Un exemple du mirage californien à ses débuts est fourni par le récit publié en 1850 par Bayard Taylor, envoyé spécial en Californie du New York Tribune. En exergue de ses " premières impressions de San Francisco ", Taylor mettait un avertissement quant à leur absolue invraisemblance :

Never have I had so much difficulty in establishing, satisfactorily to my own senses, the reality of what I saw and heard. I was forced to believe many things, which in my communication to the Tribune I was almost afraid to write, with any hope of their obtaining credence. (Taylor, 54)

Devant cette hallucination réalisée, le journaliste ne pouvait qu'exciper de sa fidélité à l'ordre du vécu, au risque de conférer malgré lui un caractère fictionnel à son récit ${ }^{3}$.

Chez Taylor comme chez d'autres auteurs, le merveilleux californien est surtout social et économique. Taylor s'étonne des " gold hunters » qui gagnent cinq dollars par jour en poussière d'or grattée à même le sol devant le US Hotel. À San Francisco, explique-t- 
il, l'esprit se voit contraint de « repousser ses vieilles notions instinctives sur la valeur et les affaires" en face d'une économie inouïe, excessive à tous égards: par l'enrichissement presque spontané que semble offrir la Californie, comme par l'accroissement exponentiel de l'échelle des prix et des valeurs. Aussi, bien qu'il annonce « l'état perpétuel d'étonnement soudain » qui animera plus tard la littérature du paysage californien (Wyatt, xvi), ce mirage financier ne fait-il guère de place au paysage. Jusque vers 1855 , en effet, les grands sites californiens n'existent pour ainsi dire pas; non parce qu'ils n'ont pas encore été découverts, mais plutôt parce qu'ils n'ont pas encore produit d'effets sociaux. Ce schéma général se vérifie pleinement dans le cas des grands arbres, dont les deux principales espèces (Sequoia sempervirens ou Coast redwood, la plus haute, et Sequoia gigantea ou Sierra redwood, la plus persistante) furent observées et décrites longtemps avant que leur "découverte» ne fasse sensation (Farquhar 1965, 31-39). Taylor ne faisait qu'une mention brève et neutre du "giant redwood» de la côte (Taylor, 97)4. En 1853, la « découverte» des géants de la Sierra emprunta donc son éclat à l'Eldorado.

7 La découverte canonique des "big trees» ou «mammoth-trees» de Calaveras fut relatée à plusieurs reprises par James Mason Hutchings, fervent défenseur et promoteur local du décor californien. Dans Scenes of Wonder and Curiosity in California (1860), Hutchings insistait à plaisir sur l'invraisemblance de l'histoire (Hutchings, 10). Voici le résumé de son récit.

8 Au printemps de 1852, un chasseur nommé Augustus T. Dowd, employé par une société minière pour fournir en gibier ses ouvriers dans la région de Calaveras, tombe en arrêt devant un arbre géant.

"Surely," he mused, "this must be some curiously delusive dream;" but the great realities standing before him, were convincing proof, beyond a doubt, that such were no fanciful creations of his imagination.

De retour au camp, en vain tente-t-il de convaincre les hommes de le suivre pour voir cette merveille : sa description est jugée « too much of a story ». Aussi a-t-il recours à la ruse. Le dimanche suivant, parti tôt en chasse, il revient en hâte, annonçant qu'il a tué un énorme ours grizzly. Les ouvriers, désœuvrés le dimanche, se laissent alors conduire jusqu'à l'arbre géant, à la vue duquel ils doivent admettre le récit de Dowd. Hutchings souligne que la "gratification " procurée par la vue de cet objet merveilleux compense leur déception d'avoir été joués. La fin du récit relate la mésaventure d'un certain Lewis. Certain de pouvoir tirer bénéfice du phénomène, celui-ci forme le projet de dénuder le tronc pour en exposer l'écorce. Un scélérat qu'il avait mis dans la confidence lui vole l'écorce. Lewis parcourt alors la Californie à la recherche d'autres spécimens comparables; mais il doit abandonner, ruiné et épuisé. Bientôt, la presse ayant diffusé la découverte, les amateurs de merveilleux («the lovers of the marvellous ») se pressent à Calaveras pour vérifier le prodige (Hutchings, 10-13).

Ce récit est à la fois un conte de fées et une allégorie de l'Eldorado. La découverte des «Big Trees » est un heureux hasard induit par l'avancée de la frontière. Le chasseur, parasite de l'économie minière, se heurte à l'incrédulité des ouvriers. Incapable de produire une représentation crédible (ou attirante) du prodige, il lui substitue une fiction d'ours géant, tout aussi improbable mais plus appétissante ("Grizzly Giant » restera le nom de l'arbre). Surtout, en conduisant les mineurs à l'objet merveilleux, il rassemble des hommes "privés de rapports sociaux", souligne Hutchings, et condamnés le dimanche à l'ennui et à la dépravation (cf. Crété, 98-102). Le thème de la gratification qui compense l'appétit déçu et sanctionne l'incrédulité rappelle la 
moralité d'un conte. Enfin, Lewis échoue par naïveté là où le chasseur avait réussi par ruse; voulant commercialiser la découverte, il est victime d'un autre parasite. Néanmoins, les découvreurs, et avec eux la Californie, seront vengés par la curiosité du monde.

Tout à l'évocation de cette première, Hutchings ne signalait pas qu'en 1854-55 d'autres larrons cherchèrent à exploiter le monstre. En 1854, l'un des plus gros spécimens du bosquet de Calaveras fut abattu à l'aide d'énormes scies hydrauliques, moyennant plus de vingt jours de travail pour cinq hommes ; l'écorce en fut dénudée et expédiée à San Francisco puis à New York, où son exposition se heurta à l'incrédulité. Peu après, cependant, un autre exemplaire fut exposé au Crystal Palace de New York, avant de rejoindre son prototype londonien (Farquhar, 85). C'est à la suite de cette révélation, confirmée par une « découverte » similaire intervenue en 1856 à Mariposa, que le «Big Tree » californien devint une curiosité mondiale - et une attraction locale, la souche du géant de Calaveras étant bientôt transformée en piste de danse.

11 C'est donc dans la mesure où le phénomène parut vendable que la découverte du séquoïa fut diffusée. La promptitude des mineurs à abattre, dénuder et taillader les géants qui seraient bientôt révérés comme des images pieuses reflétait leur pratique de la forêt comme ressource exploitable et corvéable à merci. L'exploit de Calaveras eut d'ailleurs initialement un retentissement égal à celui des statistiques du phénomène. Enfin, le récit de Hutchings illustre le déploiement rapide de l'objet séquoïa dans une société à la fois curieuse et méfiante : de l'observation sur place au prélèvement d'une trace et à son exposition, puis aux dépêches de presse et à l'afflux des visiteurs en quête de vérification... En abattant un géant, les mineurs avaient sublimé la geste de la Frontière ; mais en expédiant son écorce à New York, ils firent entrer le séquoïa dans le circuit et la logique de la représentation.

\section{Explorations et réexplorations du séquoïa : genèse et morphologie d'un genre}

Comme le rapportait en 1868 le géologue Josiah D. Whitney, la communauté scientifique fut la première à s'emparer du phénomène, pour lui chercher une appartenance et surtout une appellation. Après bien des discussions, celle qui fut finalement adoptée - Sequoia gigantea - eut, aux yeux des savants américains, le double avantage de souligner la parenté générique du redwood de la Sierra avec l'espèce côtière, précédemment baptisée Sequoia sempervirens, et d'associer ce que Whitney appelait « l'arbre le plus grand et le plus intéressant d'Amérique » à un nom indigène, celui du légendaire chef Cherokee Sequoyah, que son œuvre d'alphabétisation avait transformé en symbole de consensus 5 .

13 Cependant, ce nom de Sequoia n'avait rien de californien, et sa propagation traduisit l'emprise du langage scientifique sur l'imagination commune. C'est surtout à la suite des observations des savants sur les dimensions, l'âge et l'écologie des grands arbres que se développa dans la littérature de voyage une thématique similaire. De fait, après 1860-1865, l'exploration scientifique de l'Ouest déclencha partout un foisonnement d'entreprises individuelles d'« interprétation de la terre " (Trachtenberg, 18). C'est ainsi que les promoteurs des bosquets de Mariposa et de Calaveras vendirent bientôt aux visiteurs le droit de baptiser un spécimen et même la plaque de marbre nécessaire 
(Farquhar, 85). De même, les récits de visite aux grands arbres qui proliférèrent après 1860 popularisèrent l'exploration sous les espèces du merveilleux naturel ${ }^{6}$.

En 1862, le Français L. Simonin publia dans l'une des premières livraisons du magazine Le Tour $d u$ Monde une relation de voyage en Californie qui fit date ${ }^{7}$, et qui comprenait une description des séquoïas de Mariposa. Tout en y voyant un caprice de la nature, il en donnait assez sobrement les mesures, ainsi que des illustrations analogiques : douze à trente mètres de circonférence, soit autant d'individus pour en faire le tour ; cent cinquante mètres de hauteur, soit huit immeubles de cinq étages; quatre mille ans d'âge, ce qui faisait remonter la naissance des arbres en-deçà du déluge ${ }^{8}$. Dans un tronc évidé qui avait été reconstitué à San Francisco, ajoutait Simonin, on avait pu installer un piano et une piste de bal pour vingt personnes, ou encore un petit bazar' ${ }^{9}$. Les incrédules étaient renvoyés à l'Exposition du Crystal Palace de Londres. Venaient enfin des commentaires sur l'expression de mammoth trees et les noms attribués aux arbres dans le bosquet de Calaveras.

Plus extraordinaire est le récit que publia en 1864 l'écrivain et journaliste Fitz H. Ludlow, bohème qui avait fait sensation quelques années plus tôt en racontant ses expériences d'opiomane dans un livre intitulé The Hasheesh Eater, et qui était accompagné en Californie d'Albert Bierstadt. Ce texte expose longuement les paradoxes de la représentation. En exergue, et comme pour faire suspens dans la narration, Ludlow disserte sur les chausse-trapes de la croyance: même les moins sceptiques ne peuvent croire la vérité " littérale » sur les séquoïas; s'ils " croient » aux dimensions alléguées des arbres, c'est parce qu'ils ne s'en forment aucune "réalisation concrète »; de fait, l'auteur lui-même avait pensé "croire ", mais quand il a vu, il a compris qu'il s'était trompé en acceptant le "marché » de croyance proposé par ses informateurs. Ludlow n'en vient pas moins par la suite à renouveler ce marché de dupes, en illustrant les dimensions des arbres grâce à deux types d'analogies: des expériences sur place (gravir une échelle posée contre le tronc d'un arbre tombé à terre, creuser un trou dans son écorce, se tenir debout sur son cheval à l'intérieur du tronc creux), mais aussi de suggestives fictions. Le lecteur est invité à imaginer l'arbre tombé en face du pignon d'une maison à étage ; pour aller du toit de la maison jusqu'au sommet du tronc, il faudrait marcher sur une planche inclinée vers le haut. L'âge des plus vieux arbres, estimé à trois mille ans, est également souligné par des images frappantes, que Ludlow emprunte à Thomas Starr King: le jeune arbre poussait lors de la ruine de Troie, et avait déjà dépassé l'âge des légendaires chênes de la Nouvelle-Angleterre quand Salomon construisit le Temple. Suit un second commentaire généralisant sur l'incapacité de l'esprit à saisir concrètement des durées ("time-images») dont les dimensions excèdent l'expérience humaine. Enfin, Ludlow, qui avait observé ses compagnons peintres au travail, insiste dans la même veine dénégatrice sur l'inaptitude de l'image à fournir une représentation satisfaisante («anything like a realizing picture of the groves»). Si couleur et forme, explique-t-il, peuvent être indiquées par la peinture, la taille ne saurait l'être, même au moyen de figures à valeur d'échelle: car à moins de recourir à une toile géante, ces figures feraient l'effet d'« homoncules " placés devant des arbres de taille normale. La stratégie rhétorique de ce texte paradigmatique est éclatante : sous couvert de faire droit au phénomène, il s'agit d'en rejeter l'expérience « réalisante » dans l'ordre de l'ineffable, tout en ciselant un morceau de bravoure. 
16 Le problème délicat de l'illustration des séquoïas ${ }^{10}$ reçut cependant un traitement diamétralement opposé dans un article du révérend H. J. Morton, qui commentait en 1866 une série de photographies de la région de Yosemite pour une revue spécialisée. À cette date, la photographie californienne avait déjà atteint une sorte d'apogée, et les paysages de Carleton E. Watkins, entre autres, étaient partout admirés. Morton n'en était pourtant pas pleinement satisfait. Regrettant que les vues ne comportent pas de figures humaines - « un homme debout devant un arbre nous montre d'un coup s'il s'agit d'un arbuste ou d'un superbe pin touchant à la voûte céleste» - le révérend relatait dans une saisissante digression la déception qu'il avait éprouvée, lors de sa première visite à Saint-Pierre de Rome, quant à la taille de l'édifice ; déception corrigée par une expérience para-optique d'élargissement de l'espace, l'observateur s'étant aperçu que des figures qu'il évaluait de loin à un pied de hauteur en mesurait en réalité six : «alors le bâtiment se mit à grandir - à s'étendre de tous côtés - et sa vastitude nous fut révélée ». De même, poursuivait-il, avait-il été déçu au premier regard par une photographie du "Grizzly Giant ", avant d'apercevoir au pied de l'arbre un groupe d'hommes, dont « l'effet sur l'image fut magique » :

The tree rose and rose as we followed up its trunk (from the points where certain definite standards of height were visible), and towered aloft in majestic proportions, till at last the eye, almost wearied with the work of following its solemn shaft, took in the whole stupendous growth, and we felt that we looked indeed upon a grizzly giant.

Ainsi, Morton réagissait à l'opposé de Ludlow sur la question de l'échelle, et se montrait comblé au seul vu d'une image. L'image pouvait donc apporter une "réalisation » satisfaisante - mais elle aussi paradoxale, puisqu'elle générait à son tour du merveilleux, cette fois sur le mode magique et gullivérien de l'« expansion ${ }^{11}$.

Dans les années 1860 , les annales du séquoïa insistent plutôt sur l'inanité de la représentation, aggravée désormais par la déception du voyageur trop imprégné de lectures. En 1865, Samuel Bowles rapporte la désillusion que procure la vision directe des séquoïas : ils ne sont pas aussi grands qu'on le dit, et ne paraissent pas aussi grands qu'ils sont en réalité (Perl, 61). Le même thème apparaît chez John Todd en 1870, et dans un article du Scribner's Monthly en 1871. Dans un guide de Yosemite paru en 1868, John Hittell en avertissait les touristes.

18 Après 1870, toutefois, certains auteurs insistent plutôt sur la résolution du paradoxe, tout en montrant une distance à l'égard du côté burlesque de la séquoïamanie. I. Bromsley publie ainsi en 1872 un récit assez original, dans lequel la déception initiale trouve une solution neuve (et plus écologique que les précédentes) dans le repos et la fréquentation prolongée des arbres, dont les pouvoirs d'impression croissent avec les heures qui passent ${ }^{12}$. Puis il moque les noms de héros politiques ou militaires attribués aux plus grands arbres, épithètes dérisoires à ses yeux. Le reste de l'article n'en reprend pas moins les topoi connus : méthodes diverses pour arriver à la "réalisation » de la taille des arbres; méditation sur leur âge, débouchant sur une vision aussi mystique que convenue de ces « premiers temples de Dieu $»^{13}$.

Quant aux voyageurs français, ils se contentent souvent de reprendre le formulaire américain consacré, sauf à marquer une distance ironique par rapport à un culte qu'ils présentent parfois comme une naïveté yankee. En 1869, Rodolphe Lindau compare la hauteur des séquoïas à celle de la flèche de la cathédrale de Strasbourg ${ }^{14}$, et se borne à renvoyer les curieux à l'exposition permanente de Londres. En 1876, Th. Kirchhoff fournit pour ainsi dire la récapitulation des figures de l'irreprésentable, avant de se 
livrer à une amplification poétique qui marie tout aussi banalement l'architectural et l'épique. Comme Bromsley, il conclut par une critique des noms des arbres, ici vaguement mimologique: l'«Empereur Norton», baptisé d'après le célèbre mythomane san-franciscain, «a eu l'esprit de s'abattre »; entre les "Trois Grâces », on pourrait loger l'église de la Trinité, la plus élevée de New York. Ainsi la distance satirique débouche-t-elle in extremis sur la reconduction d'un registre ludique, sinon merveilleux.

20 À travers ces différents exemples, on distingue une configuration rhétorique uniforme, dans ses modalités comme dans sa fonction. Dans tous les cas, il s'agit dans les textes étudiés de traduire dans le code du merveilleux - familier à l'époque de Lewis Carroll le vécu d'une exploration en pays-frontière. Le bosquet de séquoïas est à cet égard un objet idéal, à la fois très localisé, facile d'accès, et productif d'un effet maximal. Quant à la surenchère à l'ineffable, elle vise à préserver, dans une littérature surchargée d'exotisme, un merveilleux naturel qui constitue alors un précieux fonds de commerce pour les magazines concernés. Cette surenchère anime particulièrement les récits, où les variations sur l'ineffable remplissent en outre une fonction dramatique de suspens, d'autant plus nécessaire que les « impressions » à communiquer sont plus rebattues. À l'intérieur de cette économie, la morphologie des descriptions de séquoïas se forme à un scénario remarquablement homogène - à un petit nombre de règles d'écriture, qui tendent à définir le séquoïa comme genre rhétorique (voir tableau en annexe).

\section{La fin d'une époque?}

21 La séquoïamanie disparaît-elle après 1900 ? Elle change au moins de statut. Le thème héroïque du "père de la forêt " sera peu à peu confiné à la littérature enfantine ${ }^{15}$. Le registre burlesque des promenades à cheval dans le tronc s'adaptera à l'automobile : l'image du Wawona Tree enjambant une route apparaît dans la publicité vers 1900 (Anderson, 277-281), et on peut aujourd'hui encore en Californie du nord « traverser un arbre " en voiture. Mais ce genre de traversée n'a plus le prix d'antan, et revêt une connotation vulgaire et anti-écologique. Car si le discours du merveilleux s'éteint à peu près vers 1900, c'est parce qu'il est alors balayé par une approche à la fois plus intime et plus sérieuse du paysage, incarnée par John Muir et ses écrits écologistes. Cette réinvention de la Nature américaine dans le Parc National est l'un des visages de la fin de la Frontière; et Turner lui-même - natif comme Muir du Wisconsin ${ }^{16}$ - rendra hommage à sa façon au moment fondateur que fut, en 1904, la visite de Theodore Roosevelt à Muir dans la Sierra, dont l'histoire veut qu'elle ait inspiré la politique de préservation des forêts : un moment, écrira-t-il, d'« école buissonnière [creative truancy] dans le cœur sauvage du Nouveau Monde » (Muir, xii).

Or, en notre fin de $\mathrm{xx}^{\mathrm{e}}$ siècle, la solennité institutionnellement associée au séquoïa n'empêche pas la résurgence d'une lecture «californienne » de la Forêt qui est une autre forme de creative truancy. Après que les beatniks, puis les hippies, enfin les golden boys, ont renouvelé le «California Dream » et l'esthétique de l'hallucination, la Californie n'a cessé d'être le territoire de l'excès, au point qu'un ouvrage psychanalytique récent en fait une sorte d'archi-syndrome adolescent: "California is that surplus of everything which begins with feeling good about oneself - begins, that is, with the body » (Rickels, 3). On se souvient que les auteurs de paradoxes sur le séquoïa furent nombreux à proposer le corps comme mesure de l'arbre, suggérant ainsi que le géant 
appelait déjà à un décentrement de la conscience vers les vibrations sensorielles. Ce n'est donc pas une simple coïncidence si deux des séries télévisées américaines les plus populaires de la période récente, Twin Peaks puis The X-Files, produites et situées sur la côte Ouest, utilisent de manière récurrente un décor de forêts sombres et inquiétantes, propices à toutes sortes d'événements paranormaux et en particulier de transmutations des corps. Dans les derniers épisodes de Twin Peaks, l'agent Dale Cooper, écologiste convaincu et poète à ses heures, franchit un rideau d'arbres derrière lequel il a précédemment identifié le repaire du sinistre Bob ; une fois passée ce que la série de David Lynch désigne très clairement comme la frontière du monde normal, Cooper paraît se muer à son tour en monstre sanguinaire, livrant les habitants de Twin Peaks à un avenir sombre et les aficionados à une interminable incertitude. Bien entendu, The $X$-Files n'apportera pas de réponse à ce mystère, pas plus qu'à aucune des énigmes qui dorment dans les archives du FBI : car la fonction cardinale de la série est précisément d'illustrer en toute occasion le slogan selon lequel « la vérité est ailleurs $»^{17}$. Quoiqu'elle puise à l'évidence dans un fonds mythique beaucoup plus vaste, la logique dramatique des séries paranormalistes des années 90 rappelle donc les jeux de langage victoriens sur le séquoïa : dans les deux cas, il s'agit d'explorer dans la forêt extrême-américaine les "frontières du réel», en affrontant tous les risques sauf celui de rassasier la curiosité du public.

\section{BIBLIOGRAPHIE}

\section{Sources citées}

ANDERSON, Nancy K. “The Kiss of Enterprise," in William H. Truettner (ed.), The West as America: Reinterpreting Images of the Frontier, 1820-1920, Washington: Smithsonian Institution Press, 1991, 268-277.

CRETE, Liliane. La vie quotidienne en Californie au temps de la ruée vers l'or (1848-1856), Paris : Hachette, 1982.

FARQUHAR, Francis P. Yosemite, the Big Trees, and the High Sierra: A Selective Bibliography, Berkeley: University of California Press, 1948.

FARQUHAR, Francis P. History of the Sierra Nevada, Berkeley: University of California Press, 1965.

HUTCHINGS, James Mason. Scenes of Wonder and Curiosity in California, San Francisco: Hutchings \& Rosenfeld, 1860.

MILLER, Angela. "The Mechanisms of the Market and the Invention of Western Regionalism: The Example of George Caleb Bingham," in David C. Miller (ed.), American Iconology: New Approaches to Nineteenth-Century Art and Literature, New Haven: Yale University Press, 1993.

MUIR, John. My First Summer in the Sierra [1911], Introduction by Galen Rowell, Boston: Houghton Mifflin, 1998. 
PERL, Jed. “The Vertical Landscape: 'In the Redwood Forest Dense'," Art in America (Jan.-Feb. 1976), 59-63.

RICKELS, Laurence A. The Case of California, Baltimore: The Johns Hopkins University Press, 1991. SCHAMA, Simon. Landscape and Memory, London: HarperCollins, 1995.

TAYLOR, Bayard. Eldorado or Adventures in the Path of Empire [1850], Introduction by Robert Glass Cleland, Lincoln: University of Nebraska Press, 1988.

TRACHTENBERG, Alan. The Incorporation of America: Culture and Society in the Gilded Age, New York: Hill and Wang, 1982.

WHITE, Richard. "Frederick Jackson Turner and Buffalo Bill," in James R. Grossman (ed.), The Frontier in American Culture, Berkeley: University of California Press, 1994.

WHITNEY, Josiah D. The Yosemite Guide-Book, Cambridge: Cambridge University Press, 1870.

WYATT, David. The Fall Into Eden: Landscape and Imagination in California, Cambridge: Cambridge University Press, 1986.

\section{ANNEXES}

\section{Morphologie du genre rhétorique « séquoïa »}

1. Les représentations apprises nuisent à l'expérience sur place :

1.1. Soit elles n'ont aucune efficacité préparatoire et sont des sous-estimations ;

1.2. Soit elles provoquent une attente excessive qui gâche la perception (surestimation).

2. Les statistiques et les données de l'expérience sont incompatibles :

2.1. Les représentations purement numériques sont trop abstraites ; les arbres n'apparaissent pas aussi grands qu'ils sont « en réalité », c'est-à-dire numériquement.

2.2. Le regard seul ne permet pas une juste appréciation numérique.

NB. Ces deux sous-propositions sont symétriques. Le regard ne procure l'étonnement que s'il est informé numériquement ; mais les nombres seuls ne « parlent » pas au regard.

3. L'expérience visuelle est souvent trompeuse, notamment quant à la taille des arbres ; la médiation par des figures à fonction d'échelle induit des effets paradoxaux (rétrécissement des figures).

4. La « réalisation » souhaitable de la taille des arbres passe par une expérience physique de leurs dimensions, laquelle requiert le mouvement et exclut la représentation :

4.1. Sur place, cette expérience suppose un mouvement du corps, seule mesure efficace des grandeurs : autour de l'arbre, à travers son tronc, le long de l'arbre couché, etc.

4.2. Un effet complémentaire est obtenu en agissant sur l'arbre, soit pour en prendre les dimensions (circonférence, épaisseur de l'écorce), soit pour en prélever un fragment. 
4.3. Si l'observateur est placé assez près de l'arbre, ou couché à son pied, le mouvement de son regard peut suffire à lui procurer cette expérience.

4.4. Par définition, ces expériences ne sont pas représentables; un substitut en est toutefois la contemplation des reconstitutions d'arbres à distance.

5. Des expériences imaginaires peuvent compléter ou suppléer ces expériences physiques, et éventuellement tenir lieu de représentations : ainsi l'insertion de séquoïas dans des scènes urbaines.

6. L'effet de la « réalisation » est une métamorphose gullivérienne des proportions de l'espace vu.

NB. Cette proposition est symétrique de (3). La figuration tend à rétrécir l'objet, la réalisation à l'agrandir.

7. Même le voyageur qui a « réalisé » sur place la tailles des séquoïas est encore incapable d'en fournir une représentation satisfaisante :

7.1 La taille ne peut être comparée qu'a des objets architecturaux : cathédrales pour la hauteur, maisons à étage pour le diamètre ;

7.2 La représentation iconique est difficile, parce que le jeu de l'échelle réduit la taille des figures humaines au lieu de suggérer la taille des arbres (contra Morton).

\section{Nomenclature des descriptions de séquoïas étudiées}

Isaac H. Bromsley. "The Big Trees and the Yosemite. The Wonders of the West I," Scribner's Monthly, vol. 3 (1872), 261-277.

John S. Hittell. Yosemite: Its Wonders and its Beauties, San Francisco: H. H. Bancroft, 1868. Théodore Kirchhoff. « Les merveilles de la vallée de Yosemiti », Le Tour du Monde, vol. XXXII (1876), 177-192.

Rodolphe Lindau. « Le chemin de fer du Pacifique », I, « De San Francisco à New York », Revue des deux Mondes (1869, $\mathrm{n}^{\circ}$ 6), 5-37.

Fitz Hugh Ludlow. "Seven Weeks in the Great Yosemite," The Atlantic Monthly, vol. 13 (1864), 739-754.

H. J. Morton. “Yosemite Valley,” The Philadelphia Photographer, vol. 3 (1866), 376-379.

J. S. “A visit to the 'Great Yo-Semite'," Scribner's Monthly, vol. 2 (1871), 396-400.

L. Simonin. « Voyage en Californie », III, « Le compté de Mariposa », Le Tour du Monde, vol. V (1862), 17-32.

\section{NOTES}

1. Parmi lesquels le peintre Albert BIERSTADT et le pasteur unitarien Thomas Starr KING

2. Sur le "rêve californien ", voir Kevin Starr, Americans and the California Dream, 1850-1915, New York : Oxford University Press, 1973 ; David Lavender, California: Land of New Beginnings, New York : Harper \& Row 1972.

3. Comme le remarquera un peu plus tard un jeune Français du nom d'Ernest Frignet dans une étude intitulée La Californie (Paris : Schlesinger, 1866) : la relation de voyage, explique Frignet, est 
en fait « une sorte de fiction» qui «choque notre raison» (p. x). Aussi s'agit-il ici de contrer l'illusion voulant qu'en Californie «tout se passe au rebours des habitudes et des usages reçus ", et de montrer que le mirage californien obéit aux ressorts universels de la civilisation.

4. Dans son second livre californien (New Pictures from California, 1894), Taylor se livra à une description approfondie, conforme au modèle que nous étudions ci-après.

5. Voir sur tout ceci Whitney, 140-141. Un botaniste anglais avait proposé le nom de Wellingtonia. Quant à l'appellation de Sequoia, on notera qu'elle fut appliquée à l'espèce côtière par un botaniste et ethnologue autrichien, et que son extension à l'espèce de la Sierra fut suggérée par la Société Botanique de France.

6. On ne saurait offrir ici qu'un échantillon de ce corpus immense et mal recensé. Plutôt que les grands textes, qui sont bien présentés par Farquhar (1948), nous avons choisi des articles plus ordinaires, parus dans des magazines américains et français. Les références de ces documents figurent en annexe. On trouvera d'autres sources pertinentes dans Schama, Anderson, ainsi que dans l'étude de Perl, qui cite notamment une pièce de théâtre consacrée aux « mammoth-trees ».

7. Blaise Cendrars faisait encore allusion à ce récit dans L'or (1922).

8. Ce thème biblique avait déjà été associé aux forêts américaines par la génération transcendantaliste (Schama, 197-201). Il devint avec la popularisation des séquoïas un lieu commun, qui apparaît par exemple en 1859 dans le récit californien d'Horace Greeley, le rédacteur en chef du New York Tribune (Anderson, 275). Après 1860, ce registre fut encore enrichi par le groupe des intellectuels san-franciscains, notamment Thomas Starr King, qui opposa le paganisme du monde gréco-romain au séquoïa comme « Hebrew tree » (Schama, 189). Voir aussi sur ce thème dans une perspective psychanalytique, les remarques d'Hubert Damisch, Skyline : la ville Narcisse (Paris : Seuil, 1996), 180-181.

9. Cette imagerie de fête et de dépense évoque le registre du carnaval, et vaut recréation fantasmatique de la frontière comme espace de transgression.

10. Celui-ci fait l'objet de l'étude de Jed Perl. Outre le problème de l'échelle, la difficulté majeure était pour les illustrateurs l'absence de perspective dégagée: "le paysage ne se "composait" jamais en une succession juste de premier plan, second plan, lointain» (Perl, 59). Les dessinateurs choisirent d'isoler artificiellement un ou plusieurs arbres, ou parfois de présenter un collage de points de vue partiels. Les photographes eurent plus de peine. Quant à Bierstadt, il n'hésita pas à recomposer radicalement le paysage, comme dans Giant Redwood Trees of California (1874).

11. Dans son récit de 1894, Bayard Taylor faisait état de ses efforts pour dessiner les séquoïas, et d'une "révélation» comparable, quoique liliputienne cette fois («The hostess and my wife standing together at the base of the tree, became the veriest dwarfs » : cité par Perl, 60). Sur cet imaginaire de la mesure, voir Pietro Bellosi, «Lilliput et Brobdingnag, Métaphores de l'imaginaire miniaturisant et mégalisant », Communications, $n^{\circ} 42$, «Le Gigantesque » (1985), p. 229-244.

12. Cette sortie du paradoxe paraît assez émersonienne, et préfigure en tout cas l'écologie esthétique de John Muir, que David Wyatt résume par la notion de "possession du paysage » (Wyatt, 32-46).

13. Bromsley cite ici l'expression alors célèbre de "God's first temples », qu'avait appliquée aux forêts américaines William C. Bryant, poète quasi officiel du paysage américain au XIX ${ }^{\mathrm{e}}$ siècle, qui eut un rôle déterminant dans la genèse du registre biblico-mystique (Schama, 198-201). En cette même année 1872, Mark Twain ironisait sur le culte genteel des séquoïas en lui opposant, comme exemple plus authentique des "grandeurs de la côte pacifique ", l'épisode fameux du voyage accéléré d'Horace Greeley dans la diligence de Hank Monk (Roughing It, chapitre 20).

14. À propos de cette image, plus rare mais logique dans un contexte imprégné par le romantisme allemand, voir Schama, 236-238. 
15. Voir par exemple l'album pour enfants de Harriet E. Weaver, There Stand the Giants, The Story of the Redwood Trees (Menlo Park : Lane Book Company, 1960).

16. John Muir est en fait né à Dunbar (Écosse) en 1838. Il n'a immigré dans le Wisconsin avec ses parents qu'en 1849, à l'âge de onze ans (note de l'éditeur).

17. Sur l'épistémologie négative de la série, voir David Lavery, Angela Hague et Marla Cartwright, eds., "Deny All Knowledge": Reading the X-Files (Syracuse: Syracuse University Press, 1996).

\section{RÉSUMÉS}

The sequoia files : l'arbre merveilleux : La vogue du séquoïa géant de la Sierra Nevada qui traverse la seconde moitié $\mathrm{du} \mathrm{XIX}^{\mathrm{e}}$ siècle atteste non seulement la valeur emblématique du «big tree » pour l'idéologie primitiviste et nationaliste américaine, mais aussi le goût plus populaire du merveilleux naturel, largement associé à la Californie après l'Eldorado. Cet article passe en revue une série de descriptions de séquoïas parues entre 1860 et 1890 , pour y repérer les principales figures d'un genre très réglé. À partir du défi à la représentation que constitue le séquoïa, cette littérature tend à décrire la logique de l'exploration en termes purement rhétoriques, et à traduire ainsi l'altérité de la frontière en un espace de jeu langagier, qui semble typique de l'époque victorienne.

The Sequoia Files: the tree of wonder: The cult of the Sierra Nevada giant sequoia, which lasted through the second half of the $19^{\text {th }}$ century, reflected not only its significance as an emblem for American primitivist and nationalist discourse, but also the more popular taste for the marvelous in nature, widely associated with California after 1850. This paper reviews a series of descriptions of sequoias published between 1860 and 1890 , in order to identify the main components of a highly consistent genre. Centering on the sequoia as a challenge to representation, this literature tends to describe the logic of exploration in purely rhetorical terms, and thus to translate the otherness of the frontier into a space of language play, which seems typical of the Victorian era.

\section{INDEX}

Thèmes : Perspectives 